ÉGYPTE monde arabe

\section{Égypte/Monde arabe}

$21 \mid 1995$

Économie égyptienne et perspectives de paix au

Proche-Orient

\title{
Table ronde : Bilan et perspectives de l'ajustement structurel en Égypte : introduction
}

\section{Françoise Clément}

\section{OpenEdition}

\section{Journals}

Édition électronique

URL : https://journals.openedition.org/ema/386

DOI : $10.4000 /$ ema.386

ISSN : 2090-7273

Éditeur

CEDEJ - Centre d'études et de documentation économiques juridiques et sociales

Édition imprimée

Date de publication : 31 mars 1995

Pagination : 129-142

ISSN : 1110-5097

\section{Référence électronique}

Françoise Clément, «Table ronde : Bilan et perspectives de l'ajustement structurel en Égypte: introduction », Égypte/Monde arabe [En ligne], 21 | 1995, mis en ligne le 08 juillet 2008, consulté le 07 juillet 2022. URL : http://journals.openedition.org/ema/386 ; DOI : https://doi.org/10.4000/ema.386

Ce document a été généré automatiquement le 7 juillet 2022

Tous droits réservés 


\title{
Table ronde : Bilan et perspectives de l'ajustement structurel en Égypte : introduction
}

\author{
Françoise Clément
}

1 Le dossier qui suit rassemble les articles rédigés par les intervenants d'une table ronde organisée par Françoise Clément, chercheur au CEDEJ. La plupart des textes, écrits spécialement par leurs auteurs en arabe pour cette table ronde, où ils ont été présentés en arabe, ont été traduits vers le français par différents traducteurs, puis édités par Françoise Clément. L'article de M. Zavajil, représentant local du FMI, parvenu en anglais est publié dans sa version originale. Les textes de Françoise Clément ont été rédigés directement en français ${ }^{1}$. Dans cette introduction et les articles qui suivent, les années fiscales (du $1^{\mathrm{er}}$ juillet au 30 juin) sont notées 1993/1994. Les années civiles ( $1^{\mathrm{er}}$ janvier au 31 décembre) sont notées 1992. Une suite de deux (ou plus) années civiles est notée 1992-1993.

Introduction

2 Le 7 mai 1994, le CEDEJ a organisé en ses murs une table ronde visant à dresser un premier bilan de la politique de stabilisation et d'ajustement structurel mise en œuvre en Égypte depuis la fin de 1990. Cette rencontre, rassemblait des chercheurs de la Banque centrale, des ministères de l'Agriculture, de l'Economie et du Commerce extérieur, de la Banque nationale, de l'Institut de planification et de la Faculté d'agriculture, et du CEDEJ. Elle accueillait également des représentants du FMI, de la Banque mondiale, de l'Aide américaine, du gouvernement égyptien, ainsi que des hommes d'affaires égyptiens et français. Les interventions, suivies de débats, ont évoqué successivement le programme de stabilisation et les réformes monétaires, financières et du commerce extérieur, les changements structurels dans l'agriculture, la restructuration du secteur public, l'attente des entrepreneurs privés, locaux et étrangers. La table ronde précédant de quelques semaines le renouvellement de l'accord avec le $\mathrm{FMI}^{2}$, les débats ont été animés, tant sur le bilan de la politique en cours, que sur le diagnostic des problèmes structurels de l'économie égyptienne et des 
remèdes à y apporter. Le présent dossier, qui rassemble sous une forme synthétique l'essentiel des interventions, tente de donner une vision d'ensemble, tant du programme d'ajustement égyptien lui-même que des différents points de vue s'exprimant en Égypte à son sujet. Certaines interventions, non rédigées, n'ont pu être publiées dans ce dossier. Notons par ailleurs que les problèmes de la comptabilité nationale égyptienne, les modifications qu'y apportent tant le FMI que la Banque Mondiale, et les délais de publication des statistiques officielles justifient certains écarts de chiffres que le lecteur pourra constater entre les différents articles. Les données de l'année fiscale 1993/94 n'ayant été publiées par la Banque centrale qu'à la fin de 1994, certains chiffres manquaient aux participants pour apprécier l'évolution de la situation économique au cours de la troisième année du programme de stabilisation. Certains textes ont été réactualisés depuis par leurs auteurs, à la lumière de ces données. Nous avons en revanche développé dans l'introduction certains points qui n'avaient pas été abordés au cours de la table ronde, mais qui resituent le débat dans son contexte.

3 Les représentants des organisations internationales, $\mathrm{du}$ gouvernement et de l'opposition égyptiens se sont entendus, au-delà des divergences d'interprétation sur les causes du phénomène, sur le fait que la mise en œuvre, par le gouvernement égyptien, d'un programme de stabilisation et d'ajustement structurel en accord avec le FMI et la Banque mondiale, découlait de la situation de crise héritée des deux décennies précédentes. Comme le rappelle Ahmad Noshî (Banque centrale d'Égypte), la chute des prix du pétrole, à partir de 1986, conduit à une crise de paiements qui met en évidence le grave endettement extérieur de l'Égypte et son incapacité à mobiliser l'épargne locale. Milan Zavajil (FMI) évoque l'ampleur de la fuite des capitaux, symptôme, selon lui, des distorsions de prix et des régulations dirigistes destinées à permettre une croissance basée essentiellement sur la consommation intérieure et l'investissement public. Comme le souligne Mahmûd Salem (Bureau technique des entreprises publiques), le système de prix administrés en vigueur jusqu'en 1990 avait largement contribué à la hausse de la consommation aux dépens de l'investissement et favorisé le développement de l'inflation et des déficits publics.

Le programme d'ajustement et de stabilisation (PAS) mis en œuvre à partir de 1991 a réussi à réduire tant les dépenses de $\mathrm{FBCF}$ (formation brute de capital fixe) publique que les subventions à la consommation, ainsi qu'à augmenter les ressources de l'État et les réserves en devises. Ce résultat a été obtenu par une politique de taux d'intérêts élevés, guidés par l'émission de bons du Trésor. Le PAS est ainsi parvenu à transformer le déficit chronique des paiements extérieurs en excédent et a fait baisser l'inflation de $20 \%$ à moins de $10 \%$. Ce succès est favorisé, en grande partie, par le contexte international exceptionnel de la crise du Golfe, qui a fait bénéficier l'Égypte d'importants transferts de capitaux privés et gouvernementaux, et lui a fait obtenir la promesse de l'annulation de la moitié de ses dettes contractées avant 1987 et le rééchelonnement du solde sur 25 ans. Il est également dû aux effets récessifs du programme de stabilisation, qui accentuent la baisse de la demande globale amorcée depuis la fin du boom pétrolier. Comme l'explique Salwa al-Antary, l'économie égyptienne, redevenue solvable, reprend le service de sa dette extérieure réduite et rééchelonnée et place une part croissante de ses actifs en devises à l'étranger, afin de pouvoir rémunérer ses dépôts locaux dans un contexte de contraction du crédit intérieur. 
Cependant, la réduction spectaculaire de la part du déficit budgétaire dans un PIB en stagnation de 17\% à 2,5\% entre Juin 1991 et Juin 1994, le rétablissement de l'excédent des paiements et l'augmentation des réserves extérieures restent des résultats fragiles, largement liés à la politique de taux d'intérêts intérieurs élevés et à des circonstances exceptionnelles. La structure de l'économie égyptienne, à l'origine des déséquilibres antérieurs, n'a pas pu être modifiée par trois années d'une stabilisation essentiellement monétaire. De fait, la hausse des recettes du budget de l'État, entre 1989/90 et 1993/94, est due, pour moitié, à celle des impôts et transferts de bénéfices de l'Autorité du canal de Suez et de l'Agence nationale du pétrole, pour un tiers, à l'augmentation des bénéfices de la Banque centrale (3,7\%), des taxes douanières (9,3\%) et des taxes à la consommation (18\%) et pour le solde, aux impôts et bénéfices transférés des autres entreprises, essentiellement publiques. Or, le développement de la collecte des taxes indirectes est limité par la récession interne, tandis que les rentrées de l'impôt sur le revenu des personnes, qui ne constituent que $2,5 \%$ des recettes du budget, tendent à diminuer depuis 1991. La poursuite de la hausse de la part des recettes budgétaires dans un PIB, lui-même croissant, nécessite, comme le souligne Milan Zavajil, une extension et un déplacement de la ponction fiscale, du commerce extérieur et la production vers les revenus et la consommation. D'autre part, le maintien du déficit budgétaire au niveau record enregistré en 1993/94 à 2,5\% du PIB, suppose que la croissance de ce dernier découle à l'avenir de l'investissement des secteurs productifs privé et public, débudgétisé depuis 1992. La croissance du PIB ne doit plus reposer sur l'investissement d'infrastructures financé par le budget, comme ce fut le cas avec les dépenses de reconstruction après le tremblement de terre d'octobre 1992 et les inondations de novembre 1994. Si le représentant du FMI déplore la hausse absolue et relative de la masse salariale des fonctionnaires, traduisant la difficulté de réduire les effectifs gouvernementaux en l'absence de croissance de l'emploi dans les secteurs productifs, il souligne les progrès réalisés dans la réduction des subventions à la consommation et dans la débudgétisation de l'investissement du secteur public manufacturier.

Le second résultat spectaculaire de la politique de stabilisation est l'augmentation des réserves extérieures d'environ 11 milliards \$ entre décembre 1990 et juin $1994^{3}$. Il est aussi fragile que la réduction du déficit budgétaire. L'essentiel de cette hausse des réserves de change, grosso modo équivalente à l'excédent des paiements cumulé entre ces deux dates, est dû à la crise du Golfe: 9 milliards $\$$ de transferts officiels (réguliers et exceptionnels) ainsi que 19,6 milliards \$ de transferts privés ${ }^{4}$ motivés par les taux d'intérêts égyptiens élevés et rendus possibles par l'unification et la libéralisation des changes en 1991. En effet, de ces 28,6 milliards $\$$ de transferts, le déficit de la balance courante (hors transferts) ne consomme que 12 milliards $\$^{5}$ pendant la même période. La stabilisation du déficit courant, (hors transferts) autour de 4 milliards $\$$, est due à la stagnation en valeur des importations, en baisse d'un milliard \$ par rapport à leur niveau de 1990/91, et à la croissance modérée de 1,5 milliard \$ (+37\%) des dépenses de services, composées aux trois quarts de paiements d'intérêts sur la dette extérieure et sur les dépôts de non-résidents, ainsi que de frais relatifs aux transferts de technologie. Les recettes d'exportation de biens chutent de près de 800 millions $\$(-20 \%)$ en quatre ans, tandis que les recettes de services s'élèvent de 1,3 milliard $\$(+17 \%)$. Cette augmentation est due pour $65 \%$ à celle des revenus de placement à l'étranger des actifs égyptiens en devises. 
7 Un débat oppose les économistes égyptiens quant à l'utilisation et au mode de placement du "trésor de guerre» que constituent les réserves internationales. Ce débat a amené Fa'iqa al-Rifa'î, directrice du Centre de recherches de la Banque centrale, à clarifier la composition de ces réserves et leur mode de placement, lors d'un colloque mentionné par l'hebdomadaire économique Al-Ahrâm al-lqtisâdî en date du 2/1/95. Salawa al-Antary (Banque nationale d'Égypte) nous en donne le détail dans son article. Il en ressort que sur 17,4 milliards \$ d'actifs en devises détenus par la Banque centrale au 30 Juin 1994, les réserves égyptiennes nettes utilisables constituent 13,3 milliards \$, dont 2,3 milliards $\$$ de réserves obligatoires appartenant aux banques égyptiennes. Les réserves nettes de la Banque centrale s'élèvent donc à 11 milliards $\$$, correspondant à 12 mois d'importation. Ayant décidé d'en consacrer les $7 / 12^{\mathrm{e}}$ au financement des importations courantes, la Banque centrale dispose d'un surplus de 4 milliards $\$$.

Ces liquidités auraient permis, selon Fa'iqa al-Rifa'î, de réduire le coût des importations, payées au comptant, de près de $15 \%$ par rapport aux paiements à crédits antérieurs. Cela signifie que la baisse de $12 \%$ du montant des importations entre 1990/91 et 1991/92 correspondrait, de fait, à une hausse en volume de ces dernières de $3 \%$. Le placement à l'étranger d'une part croissante des avoirs en devises du système bancaire égyptien, équivalent à 83\% de la dette extérieure égyptienne au 30 juin 1994 selon Salwa al-Antary, permet l'augmentation des recettes de services de la balance des paiements et la rémunération d'une part de l'excédent des dépôts sur les crédits, réduisant le besoin d'émission de bons du Trésor.

Les deux principaux résultats positifs des trois années de stabilisation dépendent donc très largement de la politique monétaire, en particulier du différentiel entre le taux d'intérêt offert sur les dépôts en livres égyptiennes (LE) et le rendement des autres formes de placement sur les marchés internationaux. Or, comme le soulignent les différents intervenants, l'émission de bons du Trésor à rendement élevé, qui a permis d'absorber avec succès le surplus de liquidités induit par l'afflux de capitaux extérieurs, est coûteuse à terme pour l'économie nationale.

Les taux d'intérêts élevés sur les dépôts en livres à court terme, déterminés par le volume et les taux des bons du trésor émis, ainsi que le plafonnement du crédit aux entreprises, ont conduit à un déplacement de l'épargne des devises vers la monnaie locale (dédollarisation). Ils ont également entraîné une forte augmentation des dépôts ${ }^{6}$, dont la part dans la masse monétaire passe de $96 \%$ à 106\% entre juin 1990 et juin 1993, pour retomber à $102 \%$ en juin 1994 . Salwa al-Antary rappelle que pour pouvoir rémunérer ces dépôts, qui atteignent 139 milliards LE en juin 1994 alors que les crédits locaux ne dépassent pas 80 milliards LE, les banques ont acquis $83 \%$ du stock de bons d'État, soit 29 milliards LE. C'est donc au budget de l'État que revient la charge de l'augmentation de l'épargne locale. En quatre ans, les intérêts de la dette publique intérieure passent de $4 \%$ à $7 \%$ du $\mathrm{PIB}^{7}$ et de $8 \%$ à $21 \%$ des dépenses budgétaires, consommant sur la période $15 \%$ de ces dernières, soit le double des intérêts de la dette extérieure. La part du crédit local dans la masse monétaire chute de 68\% en juin 1990 à $55,6 \%$ en juin 1993. L'endettement monétaire des administrations publiques est réduit de $26 \%$ grâce aux liquidités fournies par le solde net des bons du Trésor et l'augmentation des recettes budgétaires. Le secteur privé, agricole et commerçant, diminue ses engagements, avec l'augmentation du coût du crédit. Les ménages recourent à l'emprunt bancaire pour l'achat de véhicules de transport (taxis, minibus) et de biens de consommation durables à usage domestique ou professionnel, ainsi que 
pour les plus riches d'entre eux, pour l'achat de bons du Trésor ou la spéculation boursière. Le secteur public productif, débiteur, en juin 1994, d'environ 30\% des crédits bancaires, ne réussit pas, faute de liquidités, à se désendetter. Son endettement auprès des banques, qui représente en juin 1994 15\% du PIB, est, avec le problème des sureffectifs, un des principaux obstacles à sa privatisation.

11 Les organisations internationales, le gouvernement égyptien et les économistes critiques vis-à-vis du programme d'ajustement s'accordent sur la nécessité de poursuivre la baisse des taux d'intérêts, tant pour réduire l'endettement public que pour relancer l'activité intérieure. Comme le rappelle Ahmad Noshî, la diminution de l'inflation intérieure de $20 \%$ à $9 \%$, succès majeur de la stabilisation, facilite la baisse des taux nominaux à partir de février-mars $1992^{\circ}$. Milan Zavajil insiste sur la nécessité de poursuivre la réduction simultanée des taux d'intérêt et de l'inflation au niveau des taux des principaux partenaires commerciaux, par la poursuite des politiques de stabilisation. En effet, le différentiel d'inflation entre l'Égypte et ces derniers, responsable de la surévaluation de $25 \%$ de la livre relativement au dollar américain sur la période 1991-94, freine les exportations de biens et de services (transferts privés inclus), dont les recettes sont perçues en 1993/94 à $85 \%$ en dollars, tout en encourageant les importations de biens et services, dont $58 \%$ sont réglés en dollars. Le FMI prévoit une réduction rapide de l'excédent des paiements avec la reprise de la croissance et l'augmentation des importations qui en découle. Il recommande donc une "dévaluation active » de la livre vis-à-vis du dollar pour stimuler les exportations et contraindre les producteurs locaux à limiter leurs importations. Le débat sur la dévaluation de la livre, objet de désaccord entre le FMI et le gouvernement égyptien, est la principale cause du report de l'accord d'annulation de la dernière tranche de $20 \%$ de la dette extérieure actualisée ${ }^{9}$. Du côté des opposants à la dévaluation, le gouvernement égyptien redoute que cette dernière provoque une fuite des capitaux à court terme, sur le modèle de la crise mexicaine de 1994, ainsi que l'accroissement de la contrepartie en livres des paiements extérieurs, diminuant considérablement les ressources courantes propres de l'État. Les industriels égyptiens craignent l'impact de la hausse des prix des importations sur leurs coûts de production ${ }^{10}$. De l'autre côté, les partisans de la dévaluation invoquent la nécessité, pour l'Égypte, de développer ses exportations de produits non pétroliers et le biais anti-exportation induit par l'appréciation de la livre. La réduction des tarifs douaniers et la suppression des obstacles non tarifaires, conformes aux accords de l'Uruguay Round ratifiés par l'Égypte, complètent le dispositif de libéralisation du commerce extérieur, destiné, comme le souligne Milan Zavajil, à renforcer la compétitivité des produits égyptiens. Ramzi Zakî, pour sa part, réfute cet argument, rappelant que, comme par le passé, la libéralisation de l'importation peut favoriser une réallocation des capitaux en faveur de cette dernière au détriment des activités productives et d'exportation.

La réforme du système bancaire constitue le troisième volet du programme de stabilisation après la politique budgétaire et monétaire. Le système égyptien se caractérise par le quasi-monopole qu'exercent sur l'activité bancaire en Égypte les quatre grandes banques publiques (National, Misr, Alexandrie et Le Caire). En juin 1994, après 20 ans de libéralisation économique, elles détiennent encore $90 \%$ des dépôts et accordent $75 \%$ des crédits. Les organisations internationales estiment que la part du secteur public dans les crédits des banques publiques, qui a crû de $25 \%$ à $30 \%$ entre 1986 et 1990, traduisait un traitement privilégié en faveur des entreprises publiques restreignant l'accès du secteur privé au crédit. Le programme de stabilisation a donc 
imposé aux banques d'État d'appliquer aux firmes publiques les mêmes critères de risques que ceux utilisés pour les sociétés privées. Or, depuis la débudgétisation des comptes des firmes publiques en 1992, supprimant l'obligation, pour l'État, d'honorer les dettes de ses entreprises, le risque des prêts accordés au secteur public a considérablement augmenté. Paradoxalement, l'unification des critères d'octroi du crédit n'a pas empêché la part des entreprises publiques dans les crédits de l'ensemble du système bancaire d'augmenter. Entre Juin 1990 et Juin 1994, la part des sociétés publiques dans les crédits totaux croît de $24 \%$ à $30 \%$, et cette même part dans les prêts des banques publiques passe de $34 \%$ à $40 \%$. La part du secteur privé dans les crédits totaux baisse de $50 \%$ à $48 \%$, malgré la progression de sa part dans les crédits des banques publiques, de $45 \%$ à $47 \%$. Quant aux ménages, leurs parts dans les crédits totaux et publics croissent respectivement de $3 \%$ à $12 \%$ et de $3 \%$ à $7 \%$. Ces évolutions traduisent trois facteurs: d'une part, l'accumulation des arriérés des entreprises publiques, qui gonfle leur endettement sans qu'elles bénéficient pour autant de financements supplémentaires; d'autre part, l'effet dissuasif des taux d'intérêts élevés sur la demande de crédit des entreprises privées; enfin, l'effort de diversification des banques en direction des ménages et des micro-entreprises. La baisse des crédits accordés au secteur privé aurait touché en priorité les agriculteurs, les commerçants et, dans une moindre mesure, les prestataires de services. Comme le souligne Salwa alAntary, la diminution des emprunts du secteur privé est partiellement compensée par une hausse des participations directes des banques publiques au capital de certaines entreprises privées ou mixtes. Ce phénomène est néanmoins susceptible d'être inversé au cours des prochaines années. En effet, la privatisation du secteur bancaire public commence par la cession des parts de ce dernier dans les banques mixtes créées depuis 1974. Prévue par le programme d'ajustement structurel, la privatisation des banques devrait s'achever en juin 1995, conformément à la lettre d'intention présentée au FMI en août 1993. Le développement du marché des titres financiers, jusqu'à présent limité à une vingtaine d'entreprises publiques et mixtes et de caractère spéculatif, est attendu tant par les organisations internationales que par le gouvernement égyptien, comme un des principaux instruments de la privatisation des grandes entreprises publiques.

Le volet privatisation du programme d'ajustement structurel, présenté par le FMI comme une condition nécessaire au développement de l'activité du secteur privé est, comme le rappelle Ahmad Noshî, celui qui a enregistré les plus faibles réalisations ${ }^{11}$. Trois des 112 grandes entreprises désignées par le gouvernement comme privatisables ont été effectivement vendues en totalité. Le problème de l'endettement des firmes publiques, la réticence du gouvernement égyptien à accepter des réductions massives d'effectifs en période de croissance du chômage et de montée de la contestation politique des courants islamistes, ont conduit la Banque mondiale à accepter un étalement dans le temps du programme de privatisations. Les craintes, exprimées par R. Zakî, de voir «le capital étranger dominer à nouveau le destin du pays » et de permettre «à des pays tiers hostiles à la sécurité nationale et aux intérêts stratégiques de l'Égypte " d'acquérir des actifs productifs égyptiens, sont partagées, sans être formulées aussi clairement par les responsables gouvernementaux. Ces derniers privilégient, dans le processus de privatisation, les hommes d'affaires connus des pays voisins alliés, tels que l'Arabie Saoudite, la Libye et le Koweït, ou les grandes entreprises américaines, européennes ou japonaises déjà engagées en Égypte, en joint-ventures ou dans de grands projets d'infrastructure. 
14 M. Salem rappelle que la réforme du secteur public ne se réduit pas à sa privatisation, et que depuis la promulgation de la loi 203 de 1991, le programme de restructuration financière et administrative des entreprises publiques, qui contribuent à hauteur de $81 \%$ à la production nationale, a progressé, même si les résultats financiers des firmes publiques restent médiocres. Ce sont en effet les entreprises publiques et mixtes qui contribuent au développement du marché financier par l'émission d'obligations et la vente au public d'une part limitée de leur capital. Les sociétés privées, où la propriété familiale domine, sont réticentes à ouvrir leur capital au public. D. Jesse (Aide américaine; contribution non publiée dans ces pages) rappelle que les négociations engagées par le gouvernement égyptien avec la Banque mondiale dès 1987 ont abouti, en février 1988, à la constitution d'une commission d'étude de la restructuration et de la privatisation du secteur public, transformé en 1992 en Bureau technique du Secteur public des affaires (BEP), dirigé par Atif Ebeid, ministre en charge dudit secteur, après la promulgation en 1991 de la loi 230 d'autonomisation du secteur public et la restructuration des organisations publiques sectorielles en holdings multisectoriels. Par ces réorganisations institutionnelles, l'initiative des privatisations a été transférée aux holdings publics, qui ont constitué des associations de prises de participation dans le capital par les employés dans la plupart des entreprises publiques. La part du capital effectivement vendu varie de $5 \%$ à $10 \%$ selon la situation financière de l'établissement. L'US AID, pour sa part, soutient le programme de restructuration et de privatisation en menant, conjointement avec le BEP, des études de faisabilité dans les différentes branches concernées par la privatisation.

Le rôle du secteur privé dans le succès de l'ajustement structurel et les attentes de ses entrepreneurs ont occupé une place importante dans les débats, grâce aux deux contributions de M. Ghanem, représentant les associations d'hommes d'affaires égyptiens, et de D. Appia, conseiller économique et commercial français en Égypte (études non publiées dans ces pages). Une élude très documentée de la mission locale de la Banque mondiale sur les défis posés au secteur privé, présentée au forum des 9 et 10 octobre - « Développement du secteur privé en Égypte : investir dans le futur »-, dresse un panorama des obstacles actuels au développement du secteur privé. Elle définit les tâches qui sont assignées à ce dernier pour que l'ajustement structurel restaure la capacité de l'économie égyptienne à assurer simultanément une croissance réelle du PIB ainsi que l'amélioration du niveau de vie. Elle conclut enfin sur les politiques économiques à mettre en oeuvre pour la réalisation de ces objectifs. Le secteur privé égyptien est dominé par la micro-entreprise et les PME, puisqu'au dernier recensement de 1986, il était composé d'agriculteurs pour $32 \%$ de ses emplois, et que $64,85 \%$ des établissements non agricoles étaient des entreprises individuelles, que $33,7 \%$ de ces établissements occupaient de 2 à 9 personnes, et que seulement 1,45\% d'entre eux compte plus de 10 employés. Or, seuls les établissements employant plus de 10 personnes (secteur «formel» ou "structuré») et qui représentaient en 1986 seulement $22,7 \%$ de l'emploi du secteur privé, sont pris en compte dans la comptabilité nationale. En 1991/92, le secteur privé formel réalisait 61\% du PIB au coût des facteurs, répartis entre l'agriculture (16,3\% du PIB national au coût des facteurs), le commerce (14,9\%), l'industrie (9.6\%), les services sociaux (7.6\%) et le BTP (3,6\%). Il réalisait $46 \%$ de la FBCF nationale, répartis entre l'industrie $(10,8 \%)$, l'extraction et la transformation du pétrole $(11,9 \%)$, l'immobilier $(11,8 \%)$, l'agriculture $(3,6 \%)$ et l'hôtellerie-restauration $(3,2 \%)$. 
16 M. Ghanem rappelle que le secteur privé formel a, pour ses entreprises nées depuis le début de l'ouverture économique, moins de quinze ans d'âge. Reconnaissant la nécessité de l'ajustement et de la stabilisation monétaire, il regrette que des mesures d'accompagnement n'aient pas été prises pour assurer « une répartition plus équitable des sacrifices » et éviter «la chute brutale des chiffres d'affaires, l'augmentation des arriérés et l'accumulation des stocks ». Il réclame "des réformes aussi radicales en faveur du secteur privé productif que celles qui ont été conduites dans le domaine monétaire ", en particulier la suppression des comportements bureaucratiques et des réglementations contraignant l'activité du secteur privé. Il demande enfin que le secteur privé soit pleinement associé à l'élaboration de la politique économique de réforme, pour que cette dernière tienne compte davantage des problèmes et des besoins des entrepreneurs locaux. D. Appia rappelle pour sa part que les succès de la stabilisation et l'avancement $d u$ programme d'ajustement est un facteur de développement de l'investissement étranger, en ce qu'il rétablit la confiance et assure davantage de transparence. Exprimant le point de vue des investisseurs français, il demande la suppression des quotas d'intégration locale et des taxes à l'importation qui en découlent, qui imposent à l'investisseur étranger d'utiliser une part d'intrants locaux, parfois non conformes aux standards requis. Il souhaite un assouplissement des réglementations tatillonnes qui ne tiennent pas compte du fonctionnement réel du marché (durée de validité des produits), la réduction des barrières non tarifaires. Comme M. Ghanem, il demande une modification de la législation du travail et des politiques d'emploi assurant une plus grande flexibilité de la main-d'oeuvre, ainsi que la suppression des quotas de nationalité qui imposent l'emploi d'un certain pourcentage de main-d'œuvre égyptienne.

17 L'impact récessif de la stabilisation sur le secteur productif, public et privé, a été souligné par l'ensemble des participants, tant au niveau de l'activité que de l'investissement et de l'emploi. Les données du ministère du Plan publiées par la Banque centrale et reprises par R. Zakî indiquent que la stabilisation a d'abord fait chuter le taux de croissance du PIB de plus de 5\% par an entre 1987/88 et 1989/90 à $1,9 \%$ en 1991/92, puis permis une lente reprise à 2,5\% et 3,6\% les deux années suivantes. Selon les estimations du FMI et de la Banque mondiale, citées par M. Zavajil et Ahmad Noshî, la récession aurait été plus prononcée avec la même tendance à la reprise à partir de 1993/94, le taux de croissance passant successivement de $0 \%$ à $0,5 \%$ et $2 \%$ entre 1991/92 et 1993/94. La croissance aurait été tirée par le secteur privé, particulièrement les transports, le commerce, la finance et les assurances avec des taux annuels moyens de $5 \%{ }^{12}$. La croissance de la valeur ajoutée agricole, qui constituait en 1991/92 16,5\% du PIB ${ }^{13}$ et employait 32\% des actifs, avait bénéficié entre 1985/86 et $1987 / 88$ des relèvements des prix agricoles et enregistré un taux annuel moyen de près de 4\%. Elle décline à nouveau à partir de 1988/89, malgré la poursuite de la libéralisation des prix et de la commercialisation atteignant $2 \%$ en 1991/92, pour remontera $3,4 \%$ en $1993 / 94^{14}$.

18 L'industrie et l'hôtellerie (tourisme) sont, avec l'agriculture, les branches les plus touchées par la récession. La valeur ajoutée industrielle voit son taux de croissance chuter d'une moyenne de 7\% avant 1990 à 1,5\%, 2,9\% puis 4\% entre 1991/92 et 1993/94. Celte de l'hôtellerie et du tourisme, soumise aux aléas de l'état de la sécurité régionale et intérieure, subit deux graves crises en 1990/91 et 1993/94, où elle retombe en termes constants à son niveau de 1991/92. La croissance publique est tirée par les 
infrastructures et le logement, grâce aux travaux de reconstruction consécutifs au tremblement de terre de 1992 et aux inondations de 1994. Les tendances révélées par l'évolution du PIB sont confirmées par celle des investissements. Entre 1991/92 et 1993/94, l'augmentation de la FBCF publique la plus élevée concerne les infrastructures (pétrole et électricité, logement et services publics). La FBCF privée croît essentiellement dans les services (hôtellerie exceptée) et le BTP ${ }^{15}$. Au niveau de l'emploi $^{16}$, la croissance annuelle de la population active ralentit entre 1989/90 et 1991/92, passant de 2,9\% à 1,6\%, Elle s'accélère à nouveau en 1992/93 pour atteindre $3,2 \%$ en 1993/94. En contrepartie, le taux de chômage augmente de $7.6 \%$ en 1989/90 à $10,1 \%$ en 1992/93, pour retomber à 9,8\% en 1993/9417.

19 La libéralisation de l'agriculture, qui occupait en 1991/92 le tiers de la population active employée égyptienne et fournissait $27 \%$ de la valeur ajoutée du secteur privé ${ }^{18}$, a été l'objet d'une attention particulière. Cette branche d'activité, qui ne dispose pas de groupes de pression et d'institutions représentatives autonomes, contrairement à l'industrie et aux services, bénéficie en contrepartie de l'intérêt des centres de recherche et des universités en Égypte. M. M. Mansûr, directeur du Centre d'études économiques du Ministère de l'Agriculture, et M. M. 'Abd al-Azîm, chercheur dans ce même centre, ainsi que M. M. Abu Mandûr, directeur du Centre de recherche en économie agricole de la Faculté d'agriculture de l'Université du Caire, ont dressé un panorama contradictoire de l'impact des reformes économiques sur l'agriculture en Égypte. Les trois intervenants ont convenu du fait que la politique de tarification des produits, des intrants et du crédit agricole, qui prévalait pendant les années 70 et 80, a induit un biais défavorable à la production et à l'investissement, occasionné des gaspillages de ressources importants, augmentant les coûts de production et décourageant les exportations. M. Mansûr rappelle que le relèvement progressif des prix d'achat des produits agricoles à partir de 1986, plus rapide que le retrait des subventions aux engrais, pesticides et semences ainsi que des subventions au crédit, a permis une augmentation du revenu agricole réel. Cette hausse du revenu réel a favorisé l'augmentation des rendements et de la production, relevant les taux d'autosuffisance des principales cultures alimentaires : blé, maïs, riz. L'accélération du rythme de réduction des subventions à partir de 1990, avec la mise en place du programme de stabilisation et d'ajustement, serait responsable de la baisse du revenu réel, dont la progression resterait néanmoins positive en 1982/93. Outre le système des prix, la petite taille et l'extrême morcellement des exploitations sont des causes de gaspillage des équipements, des intrants, de l'eau et de la main-d'œuvre, accroissant les coûts de production et la pollution des sols et de l'air. Faute d'expérience, les agriculteurs appliquent des procédés de manutention et de stockage qui rendent leurs produits inaptes à l'exportation. La pollution des sols dans la vallée du Nil, liée à l'utilisation intensive d'engrais et de pesticides, fait obstacle à l'exportation des fruits et légumes. M. Abu Mandûr souligne la nécessité d'opérer un remembrement susceptible de stopper le processus de morcellement des exploitations, permettant d'améliorer les techniques de production et de réduire les gaspillages. De fait, la concentration de la propriété foncière semble devoir se réaliser, par le jeu du marché, sous l'effet de la suppression des subventions et de la libéralisation des fermages. La conquête de 2,7 millions de feddans $\left(11270 \mathrm{~km}^{2}\right)$ sur le désert entre 1952 et 1993 est, avec la libéralisation des prix, la mécanisation et l'introduction de semences à hauts rendements, un des principaux facteurs de développement de l'agriculture égyptienne. Dégagées des contraintes foncières et des traditions de la Vallée, avec des sols non 
encore pollués, les exploitations des nouvelles terres utilisent les techniques de production et de conditionnement les plus modernes, et sont plus aptes à produire pour l'exportation. Dans la Vallée, au contraire, la pression démographique et l'absence d'emplois alternatifs fait obstacle à l'amélioration des techniques de production. La libéralisation des prix et des circuits de commercialisation induite par le programme d'ajustement, si elle ne suffit pas, comme le souligne M. Abu Mandûr, à laire croître la production locale et les exportations, est de nature à réduire les gaspillages d'intrants et les facteurs de pollution, tout en imposant aux producteurs une rationalisation de leurs méthodes de production.

\section{NOTES}

1.) Je tiens à remercier Mmes F. al-Rifâ'i et Salwa al-Antary, ainsi que MM. M. Zavajil. Ahmad Noshî, S. Azîz, J. de Lajugie. Cousseran, Ph. Fargues et F. Ireton pour leur aide et leurs conseils. Les informations et idées exprimées dans ce dossier relèvent toutefois de la seule responsabilité de leurs auteurs.

2.Égypte/Monde arabe $\mathrm{n}^{\circ} 20$, Chronique économique, p. 300.

3.DTS (droits de tirage spéciaux) et position auprès du FMI non compris. International Financial Statistics, FMI décembre 1994, pp. 214-215, et Banque centrale d'Égypte, Rapport annuel 1993/94. 4.Parfois abusivement appelés " remises des émigrés égyptiens ", les transferts privés incluent ces dernières, ainsi que les remises des émigrés soudanais dont les familles résident en Égypte, et les placements à court terme des ressortissants des pays voisins (Libye et pays du Golfe essentiellement) ou des Égyptiens ayant placé leur épargne antérieurement à l'étranger. 5.Banque centrale d'Égypte, rapports annuels 1987/88 à 1993/94, ainsi que les chiffres et pourcentages cités par la suite dans cette introduction, sauf mention d'une autre source. 6.Nous avons pris en compte la totalité des dépôts (administrations publiques inclues) dont la part dans le PIB croît de 87\% à 93\% entre juin 1990 et Juin 1993, pour retomber à 87,5\% en juin 1994, suite à la baisse des taux d'intérêts à partir de janvier 1994.

7.On dispose de deux estimations du PIB en livres courantes. La première est publiée par International Financial Statistics, op. cit., pp. 216-217, sur la base des chiffres communiqués au FMI par le Ministère du Plan, non publiés en Égypte. La seconde provient des estimations de la mission locale du FMI sur la base des données publiées en livres constantes par le Ministère du Plan et déflatées de l'indice des prix à la consommation urbaine, considéré comme estimation minimale du taux d'inflation. Cette seconde estimation donne un PIB courant légèrement inférieur à celui donné par la première, dont le taux de croissance est plus conforme aux estimations du FMI et du gouvernement égyptien que celui déduit des chiffres publiés par l'International Financial Statistics (FMI).

8.Les taux d'intérêts sur les dépôts en livres à trois mois passent de 7,5\% en 1989 à $13.6 \%$ en juin 1991 , atteignent $17,2 \%$ en janvier 1992, puis diminuent à nouveau pour retomber à $10.8 \%$ en juin 1994. Les taux d'intérêts sur les crédits à moins d'un an passent de $14 \%$ en 1989 à 19,5\% en Janvier 1991, atteignent 21.2\% en mars 1992, puis diminuent pour retomber à 16,6\% en juin 1994.

9.Cf. Chronique économique dans ce numéro. 
10.Égypte/Monde arabe $\mathrm{n}^{\circ} 20$, Chronique économique, p. 301,

11.Égypte/Monde arabe $\mathrm{n}^{\circ} 8,9,11,12 / 13,14,15 / 16,17,18 / 19$, Chroniques économiques.

12.Données du Ministère du Plan, Revue annuelle de la BCE 1992/93 et 1993/94, Annexe statistique, tableau 1 /I

13.19\% selon les statistiques du Ministère du Plan, série en livres constantes 1986/87 (Rapport annuel de la BCE 1991/92), citées par M. Mansûr.

14.Données du Ministère du Plan reprises par « Private Sector Development in Egypt : the Status and the Challenges ", Mission résidente au Caire de la Banque mondiale, octobre 1994, pour les évolutions jusqu'en 1991/92, et par les Rapports annuels de la BCE 1992/93 et 1993/94 pour les années suivantes.

15.Données du Ministère du Plan, Revue annuelle de la BCE 1992/93 et 1993/94, Annexe statistique, tableau $1 / 3$.

16.Les données de l'enquête trimestrielle « main-d'œuvre par échantillon », seule statistique disponible en Égypte sur l'évolution de l'emploi entre deux recensements, comportent des marges d'erreur d'autant plus importante que les données sont désagrégées. De plus, en 1992, l'enquête enregistre une baisse de $10 \%$ de l'emploi total, 1,5 million d'actifs étant subitement devenus chômeurs. Cette évolution contredit les données du Ministère du Plan, qui note pour la même année une hausse de $1.6 \%$ de la population active employée.

17.Données du Ministère du Plan, Revue annuelle de la BCE 1990/91,1992/93 et 1993/94, Annexe statistique tableau $1 / 4$.

18.Données du Ministère du Plan reprises par « Private Sector Development in Egypt: the Status and the Challenges ", Mission résidente au Caire de la Banque Mondiale, octobre 1994, pour les évolutions jusqu'en 1991/92.

INDEX

Mots-clés : ajustement structurel, économie

\section{AUTEUR}

\section{FRANÇOISE CLÉMENT}

CEDEJ 